\title{
MicroRNA-137 dysregulation predisposes to osteoporotic fracture by impeding ALP activity and expression via suppression of leucine-rich repeat-containing G-protein-coupled receptor 4 expression
}

\author{
XIANGJUN LIU and XIAOHUI XU \\ Department of Orthopedics, The People's Hospital of Huangdao, Qingdao, Shandong 266400, P.R. China
}

Received December 28, 2016; Accepted February 20, 2018

DOI: $10.3892 / \mathrm{ijmm} .2018 .3690$

\begin{abstract}
Osteoporosis is defined as a loss of bone mass and deterioration of its architecture resulting in bone weakness, which becomes prone to fracture. The objective of this study was to investigate the molecular mechanism by which miR-137 can reduce the risk of fracture in patients with osteoporosis. An online miRNA database and a luciferase reporter assay system were used to confirm that leucine-rich repeat-containing G-protein-coupled receptor 4 (LGR4) was the target of miR-137. Real-time PCR and western blot analysis were used to study miR-137 mRNA, the expression of LGR4 mRNA and protein among different groups or cells transfected with a scrambled miRNA control, miR-137 mimic, LGR4 siRNA and miR-137 inhibitor. Expression of miR-137 was upregulated to higher levels in cells isolated from osteoporosis patients with fracture than in those without fracture. The 'seed sequence' was found to be located within the 3 ' untranslated region (3'-UTR) of LGR4 mRNA by searching an online miRNA database. Luciferase reporter assay was performed to confirm that LGR4 is a direct target gene of miR-137 with a potential binding site in the 3'UTR of LGR4. Luciferase activity of cells transfected with wild-type LGR4 3'UTR was much lower than that of the cells transfected with mutant LGR4 3'UTR. The results of real-time PCR and immunohistochemistry experiments demonstrated that the expression levels of LGR4 mRNA and protein were much higher in osteoporosis patients with fracture than osteoporosis patients without fracture. We found that the expression levels of LGR4 mRNA and protein were clearly upregulated following transfection with miR-137 inhibitor, while noticeably downregulated following transfection with miR-137 mimic when compared with the scramble control.
\end{abstract}

Correspondence to: Dr Xiaohui Xu, Department of Orthopedics, The People's Hospital of Huangdao, 2999 Lingshanwan Road, Huangdao, Qingdao, Shandong 266400, P.R. China

E-mail: osteoporosislgr4@126.com

Key words: microRNA-137, osteoporotic fracture, ALP, leucine-rich repeat-containing G-protein-coupled receptor 4
Furthermore, the expression of ALP mRNA and ALP activity in bone tissue were much higher in osteoporosis patients with fracture than those without fracture. In conclusion, these data prove that the overexpression of miR-137 was associated with an altered risk of fracture in patients with osteoporosis, and can be used as a biomarker for the prediction of risk of fracture in osteoporosis.

\section{Introduction}

A major priority for public health authorities is to manage the effects of osteoporosis. Nearly a third of women over the age of 50 are affected by the disease, around $50 \%$ of whom have a combined lifetime risk of forearm, vertebral or hip fracture, approximately the same risk that is related to cardiovascular disease (1). In Europe, the estimated total direct cost of osteoporosis was $€ 31.7$ billion in 2000 and likely to have nearly doubled by 2050 due to demographic changes in the population (2). An osteoporotic fracture will by experienced by $\sim 50 \%$ of women and $22 \%$ of men over the age of 50 in their lifetime (3). The weakness of bone associated with osteoporosis may increase the incidence of fractures in the elderly aged 50 and above. The incidence of osteoporosis is increasing in the aging society (4).

There is an awareness that there is an interaction between certain 'lifestyle-related diseases', such as chronic kidney disease, hypertension or type 2 diabetes with osteoporosis (5). Furthermore, it has been shown that certain lifestyle-related diseases (e.g., atherosclerosis and type 2 diabetes) increase fracture risk while being unrelated to changes in bone mineral density (BMD) (6). It is believed that increased oxidative stress contributes to the association between bone metabolism and lifestyle-related diseases, by promoting the fragility of bone in patients through accumulation of advanced glycation products in bone through various mechanisms, such as abnormalities in collagen cross-link formation in bone and decreased osteoblast differentiation resulting in increased death of osteoblasts or osteocytes (6).

The main feature of osteoporosis is low BMD which is a highly heritable trait with heritability of 0.5-0.8 (7). Osteoporotic fracture $(\mathrm{OF})$ also has moderate heritability ranging from about 0.5 to 0.7 and thus acts as an end-point clinical 
outcome of osteoporosis (8). We now know that genome-wide association studies (GWASs) and their meta-analyses have identified over 20 genes/loci related to risk of osteoporosis and more than 60 related to variations in BMD. In addition, a novel rare nonsense mutation within the gene leucine-rich repeatcontaining G-protein-coupled receptor 4 (LGR4) strongly related to low BMD and $\mathrm{OF}$ has been identified in a recently published study on whole-genome sequencing.

MicroRNAs (miRNAs), a class of non-coding RNAs 19-25 nucleotides in length, control the expression of genes at the post-transcriptional level (9). The first miRNA to be discovered, lin-4, was found in Caenorhabditis elegans in 1993, and since then a number of miRNAs have been identified in different organisms (10). The latest miRBase release (v20, June 2013) reported that 24,521 miRNA loci had been discovered from 206 species producing 30,424 mature miRNA products. Numerous miRNAs regulate different pathophysiological events such as organogenesis, apoptosis, tumorigenesis, proliferation, organ development and hematopoietic function (11).

A recent in vitro study found that osteoclastogenesis in human circulating mononuclear cells was inhibited by miR-146a (12). Furthermore, another in vitro and in vivo study showed that osteoclastogenesis in human circulating mononuclear cells was promoted by miR-148a (13). A further study found higher levels of miR-133a in circulating monocytes in vivo in postmenopausal women with low BMD than in those with high BMD, and thus miR-133a can be identified as a potential biomarker related to postmenopausal osteoporosis (14). Recently, an analysis of miRNAs from total bone tissue comparing osteoporotic vs. non-osteoporotic bone was conducted $(15,16)$. Seeliger et al and Garmilla-Ezquerra et al identified six miRNAs that were upregulated in osteoporotic fracture patients: miR-21, miR-23a, miR-24, miR-25, miR-100 and miR-125b $(15,16)$. LGR4 has been shown to be associated with the development of osteoporotic facture, and the differential expression of miR-137 has been identified in the disease (16-18). LGR4 was also found to be a potential target of miR-137 in an online miRNA database. In this study, we investigated the relationship between miR-137 and LGR4 and explored their roles in the pathogenesis of fracture in patients with osteoporosis.

\section{Patients and methods}

Sample collection. A total of 30 osteoporosis patients with OF $(n=16)$ or without OF $(n=14)$, and 18 patients without osteoporosis at Department of Orthopedics, The People's Hospital of Huangdai (Qingdao, China) were included. Patients with metabolic bone disease, who had undergone therapies such as hormone replacement therapy, calcitonin, bisphosphonates and fluoride were excluded from the study. Information of the participants, such as sex, age at diagnosis, meal preferences (meat vs. vegetables), physical activity, and previous disease history were collected prior to study. The Institution's Ethics and Research Committees approved this study. The patients signed informed consent for participation in the study. The study was conducted according to the Declaration of Helsinki. All specimens were obtained after surgical resection.
RNA isolation and real-time PCR. TRIzol (Invitrogen, San Diego, CA, USA) reagent was used to extract the total RNA from cultured cells and tissue samples in accordance with manufacturer's instructions. NanoDrop ND-2000c (Thermo Fisher Scientific, Inc., Wilmington, DE, USA) was used to determine the concentrations and purities of RNA extracted. Spot Check Nucleic Acid Quantitation kit (Sigma-Aldrich, St. Louis, MO, USA) and gel electrophoresis were used to test the integrity of RNA following standard protocol. TaqMan MicroRNA reverse transcription kit (Applied Biosystems, Foster City, CA, USA) was used to reverse transcribe the RNA to DNA (cDNA) with carefully designed primer purchased from Applied Biosystems in accordance with the protocol by supplier. TaqMan hsa-miR-137 amplification kit (Applied Biosystems) was used to amplify the cDNA in accordance with manufacturer's instructions. Roche LightCycler 480-II real-time thermal cycler (Roche, Basel, Switzerland) was used to perform the quantitative RT-PCR analysis of miR-137 expression according to the manufacturer's recommendation, and the real-time PCR were performed using microRNA specific probes and TaqMan Universal Master Mix (Applied Biosystems) according to the manufacturer's instructions. RNU43 was used as the internal control to normalize the expression of LGR4 mRNA. $\Delta \Delta \mathrm{Ct}$ method was used to analyze the expression of LGR4 mRNA and miR-137. All experiments were run 3 times.

Cell culture and transfection. RPMI-1640 medium (Hyclone, Logan, UT, USA) containing 10\% fetal bovine serum (FBS), antibiotics, $1 \%$ streptomycin-penicillin and L-glutamine, was used to incubate U-2 and MC3T3 cells in a humidified atmosphere $5 \% \mathrm{CO}_{2} / 95 \%$ air at $37^{\circ} \mathrm{C}$ at a final concentration of $1 \times 10^{6}$ cell $/ \mathrm{ml}$ for $72 \mathrm{~h}$ according to the manufacturer's instructions. After the confluence reached $80 \%$, Lipofectamine 2000 (Invitrogen Life Technologies, Carlsbad, CA, USA) was used to transfect 20 pmol of miR-137 mimics or its inhibitors with U-2 or MC3T3 cells according to the manufacturer's description. The test was carried out in triplicate.

ALP activity determination. ALP activity determination kit (Abcam, Boston, MA, USA) was used to measure the ALP activity in the bone tissue samples.

Luciferase assay. The fragment 3' untranslated region (3'UTR) of LGR4 amplified by PCR was inserted into the multiple restrictive sites of psiCHECK-2 vector (Promega, Madison, WI, USA), and site-directed mutagenesis kit was used to introduce the mutation to replace the identified binding sites in the 3'UTR of the LGR4.

Lipofectamine 2000 (Invitrogen Life Technologies) was used to transiently transfect the U-2 cells with pRL Renilla as the control vector (Promega) and the promoter/luciferase reporter gene in accordance with the manufacturer's instructions, and the pRL Renilla as the control vector (Promega) was used to correct transfection efficiency. The cells were collected $48 \mathrm{~h}$ after transfection, and phosphate-buffered saline (PBS) was used to wash the cells, and then the lysis reagent (Promega) was used to lyze the cells in accordance with the manufacturer's instruction. Luciferase assay reagent (Promega) was used to detect the firefly luciferase activity, 
Stop \& Glo reagent (Promega) was used to detect the Renilla luciferase activity based on the standard by supplier. Three independent experiments were carried out.

Western blot analysis. RIPA buffer (Invitrogen Life Technologies) was used to extract the whole protein from U-2 cells in accordance with manufacturer's instruction. XCell SureLock Mini-Cell electrophoresis system (Invitrogen Life Technologies) with 12\% SDS-PAGE was used to separate the total protein, and then transferred to the polyvinylidene fluoride membranes (Immobilon-P membrane; Millipore, Billerica, MA, USA). Ponceau S staining was used to verify the equal loading of proteins. Tris-buffered saline-Tween-20 (TBS-T) with 5\% dried milk (Abcam, Cambridge, UK) was used to block the membranes, and the anti-LGR4 and anti-ALP antibodies at a dilution of 1:500 and anti- $\beta$-actin at a dilution of 1:5,000 (all from Santa Cruz Biotechnology, Inc., Santa Cruz, CA, USA) were used to incubate the membrane at $4^{\circ} \mathrm{C}$ overnight in accordance with the manufacturer's recommendation, and then the membrane was washed, the secondary antibody anti-rabbit $\operatorname{IgG}$ with a 1:10,000 dilution (Molecular Probes, Eugene, OR, USA) was used to detect the primary antibody for another $1 \mathrm{~h}$. LI-COR Odyssey Blots scanner (LI-COR, Lincoln, NE, USA) was used to visualize the corresponding fluorescence signal in accordance with the manufacturer's instructions. Anti- $\alpha$-tubulin (DM1A; Abcam) at a dilution of 1:1,000 was used to assess the equal protein loading. Odyssey 2.1 software (LI-COR) was used to calculate the quantification of protein. The band intensities of LGR4 and $\alpha$-tubulin ( $\alpha$ TUB) were corrected by subtracting the respective background intensities. All experiments were performed 3 times.

Immunohistochemistry. PBS with 4\% paraformaldehyde was used to fix the bone tissue samples, and xylenes were used to deparaffinize the sections, which were next dehydrated by a series of graded ethanol. Subsequently, hematoxylin and eosin (H\&E) was used to stain the section. The histological score of each slide was evaluated.

Statistical analysis. All data are shown as mean \pm standard deviation (SD). One-way analysis of variance (ANOVA) and Student's t-test were used to compare between the groups. SPSS version 17.0 (SPSS Inc., Chicago, IL, USA) was used to analyze the statistical procedures.

\section{Results}

miR-137 is a potential biomarker for the development of fractures. Real-time PCR was used to find potential biomarkers for the development of a fracture. We recruited 48 participants for our study, 18 osteoporosis patients with fracture, 16 without fracture and 14 in the normal group. As shown in Fig. 1, the level of miR-137 expression was much lower in the group of osteoporosis patients with fracture compared with those without fracture or the normal group, while the level of miR-137 expression was significantly higher in the normal group than in osteoporosis patients without fracture, indicating that downregulation of miR-137 expression contributes to the formation of osteoporosis and fracture.

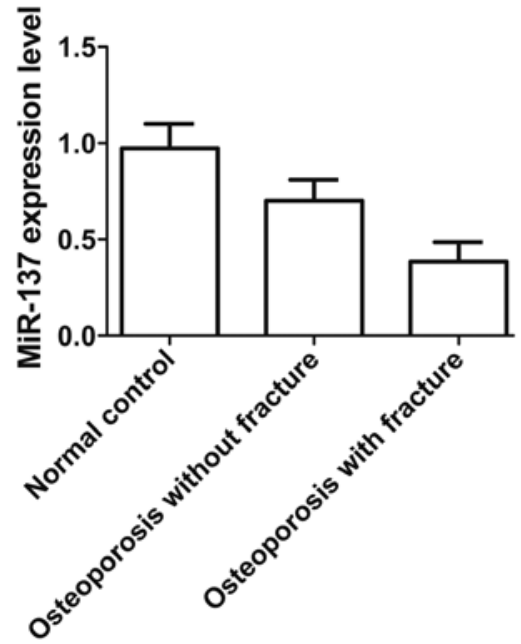

Figure 1. Expression of miR-137 was downregulated in cells collected from patients with fracture compared with osteoporosis patients without fracture and normal groups.

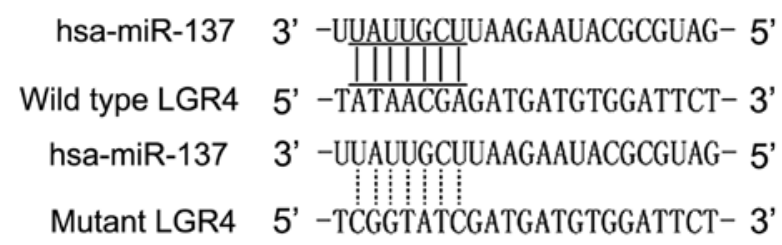

Figure 2. Leucine-rich repeat-containing G-protein-coupled receptor 4 (LGR4) was identified as target of miR-137 with possible binding regions which accordingly generated mutant LGR4 where the possible binding site sequences were replaced with their complementary sequences, respectively.

LGR4 is predicted to be a target of $m i R-137$. $\mathrm{miR}-137$ was found to be involved in the pathogenesis of various human diseases, such as osteoporosis and fracture. Based on computational analysis using the online target predicting tool TargetScan (www.targetscan.org), we identified that miR-137 was able to bind to the 3'UTR of LGR4, indicating this gene may be a potential molecular target for miR-137, as shown in Fig. 2.

LGR4 was confirmed to be a direct target of miR-137. To verify that miR-137 and LGR4 interact with each other, we performed a luciferase assay to confirm that LGR4 is a direct shared target of miR-137. As shown in Fig. 3, the luciferase activity of cells transfected with wild-type LGR4 was significantly downregulated compared with cells transfected with mutant-type LGR4 and scramble control, while the luciferase activity in the cells transfected with mutant 3'UTR of LGR4 was clearly upregulated compared with the scramble control, confirming that LGR4 is a direct target of miR-137.

Interaction between miR-137 and LGR4 mRNA. To further confirm the regulatory relationship between miR-137 and LGR4 mRNA in different cell lines, we transfected miR-137 mimic and its inhibitor into cultured U-2 and MC3T3 cells. The results of qRT-PCR analysis (Fig. 4A) revealed significantly enhanced LGR4 mRNA expression in U-2 cells transfected with miR-137 inhibitor and reduced LGR4 mRNA expression in the miR-137 mimic treatment group compared with the scramble control. The expression of 


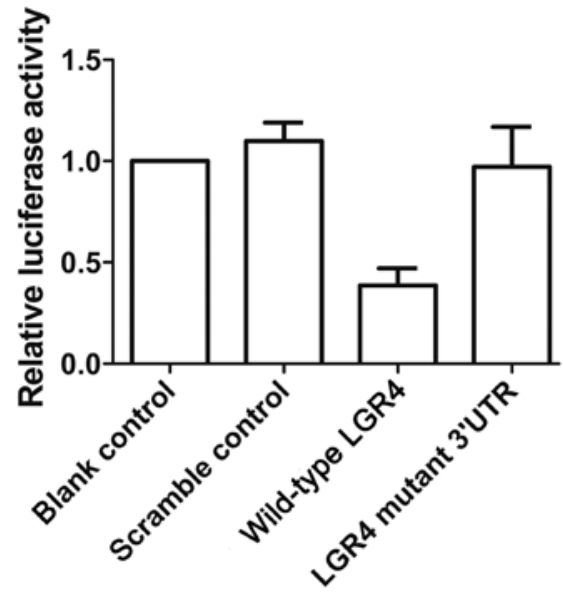

Figure 3. Luciferase activities of cells transfected with wild-type leucine-rich repeat-containing G-protein-coupled receptor 4 (LGR4) 3' untranslated region (3'UTR) was much lower than scramble and mutant1 LGR4, while notably higher when the cells were transfected with mutant LGR4.

LGR4 mRNA in MC3T3 cells transfected with miR-137 mimic was also clearly downregulated compared to that of the scramble control, and transfection with miR-137 inhibitor was also much higher than that of the scramble control, establishing the downregulatory effect of miR-137 on the LGR4 gene. The inhibitory effect of miR-137 was stronger in U-2 cells.

Interaction between miR-137 and LGR4 protein. To further confirm the regulatory relationship between miR-137 and LGR4 protein in different cell lines, we transfected miR-137 mimic and inhibitor into cultured U-2 and MC3T3 cells. The results of western blot analysis (Fig. 5A) revealed a significantly enhanced LGR4 protein level in U-2 cells transfected with miR-137 inhibitor and reduced LGR4 protein level in the miR-137 mimic treatment group compared with the scramble controls. As shown in Fig. 5B, the level of LGR4 protein in MC3T3 cells transfected with miR-137 mimic was clearly downregulated in comparison with the scramble control, and that of MC3T3 cells transfected with miR-137 inhibitor was much higher compared with the scramble control, demonstrating the downregulation of LGR4 gene by miR-137. The inhibitory effect of miR-137 was stronger in U-2 cells.

Expression level of LGR4 $m R N A$ and protein varied in different groups. Real-time PCR and immunohistochemistry were used to detect the expression level of LGR4 mRNA and protein among osteoporosis patients both with and without fracture and a normal group of patients. As shown in Fig. 6, the expression of LGR4 mRNA (Fig. 6A) and protein (Fig. 6B) were much lower in osteoporosis patients without fracture and the normal group compared with osteoporosis patients with fracture. The expression of LGR4 mRNA (Fig. 6A) and protein (Fig. 6B) were significantly lower in the normal group, suggesting that upregulation of LGR4 mRNA and protein expression may contribute to the formation of osteoporosis and fracture.

Expression level of $A L P$ and $A L P$ activity varied in different groups. Finally, ELISA was used to measure the expression
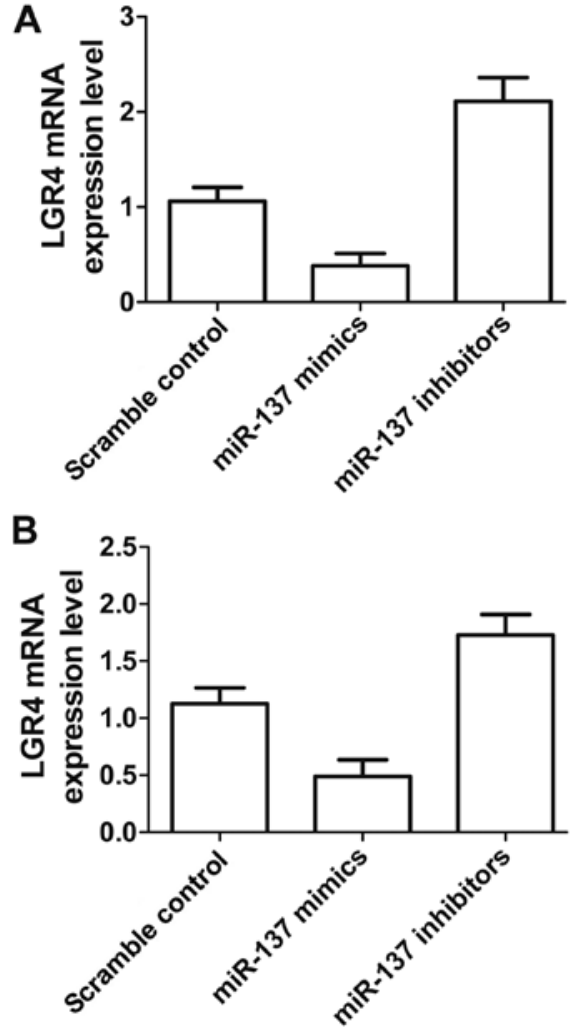

Figure 4. Real-time PCR was used to detect the effect of miR-137 on leucine-rich repeat-containing G-protein-coupled receptor 4 (LGR4) mRNA in different cell lines. miR-137 mimics, and miR-137 inhibitors were transfected into cultured U-2 and MC3T3 cells. (A) The expression of LGR4 mRNA of U-2 cells transfected with miR-137 mimics was much lower than scramble control, while the LGR4 mRNA level of U-2 cells transfected with miR-137 inhibitors was much higher than scramble control. (B) The expression of LGR4 mRNA of MC3T3 cells transfected with miR-137 mimics was much lower than scramble control, while the LGR4 mRNA level of MC3T3 cells transfected with miR-137 inhibitors was much higher than scramble control.

level of ALP and to detect ALP activity in osteoporosis patients with and without fracture, and the normal group. As shown in Fig. 7, ALP expression (Fig. 7A) and ALP activity (Fig. 7B) in bone tissue were much higher in osteoporosis patients both with and without fracture than the normal group, while both ALP expression and ALP activity were highest in osteoporosis patients with fractures, significantly so, indicating that upregulation of ALP and ALP activity contributes to the formation of osteoporosis and fracture.

\section{Discussion}

It has been shown in recent studies that miR-137 influences the process of tumorigenesis. For example, it was shown that the sensitivity of pancreatic cancer cells to chemotherapy, invasion and growth are modulated by miR-137 (19). It was also revealed that miR-137 inhibited proliferation of melanoma cells by targeting PAK2 and was considerably downregulated in melanoma (20). Moreover, it was shown that miR-137 in colorectal cancer tissues was reduced and that colony formation, tumorsphere and cell growth of colon cancer cells were inhibited by miR-137 through targeting Musashi-1 (21). It was also found that miR-137 in non-small cell lung cancer (NSCLC) tissues was downregulated and 
A
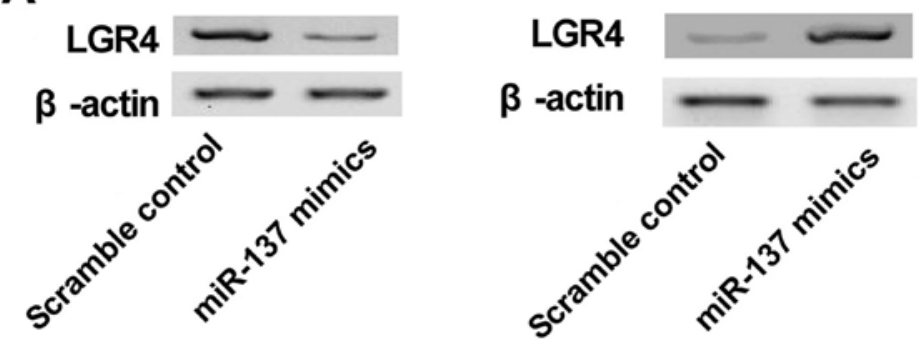

B
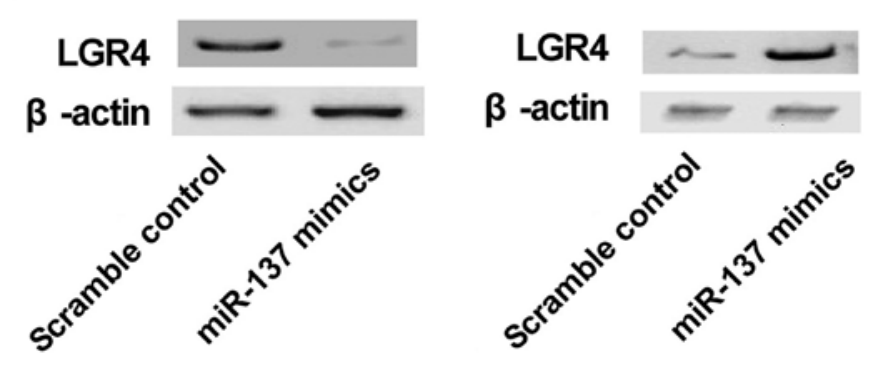

Figure 5. Western blot analysis to detect the effect of miR-137 on leucine-rich repeat-containing G-protein-coupled receptor 4 (LGR4) protein in different cell lines. miR-137 mimics, and miR-137 inhibitors were transfected into cultured U-2 cells and MC3T3 cells. (A) The expression of LGR4 protein of U-2 cells transfected with miR-137 mimics was much lower than scramble control, while the LGR4 protein level of U-2 cells transfected with miR-137 inhibitors was much higher than scramble control. (B) The expression of LGR4 protein of MC3T3 cells transfected with miR-137 mimics was much lower than scramble control, while the LGR4 protein level of MC3T3 cells transfected with miR-137 inhibitors was much higher than scramble control.

A

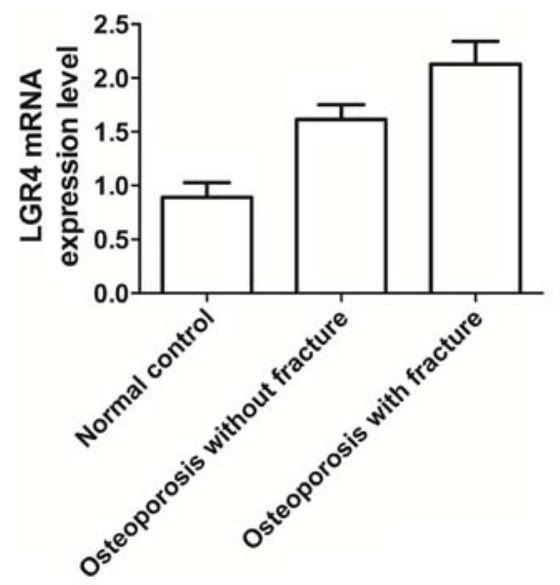

B

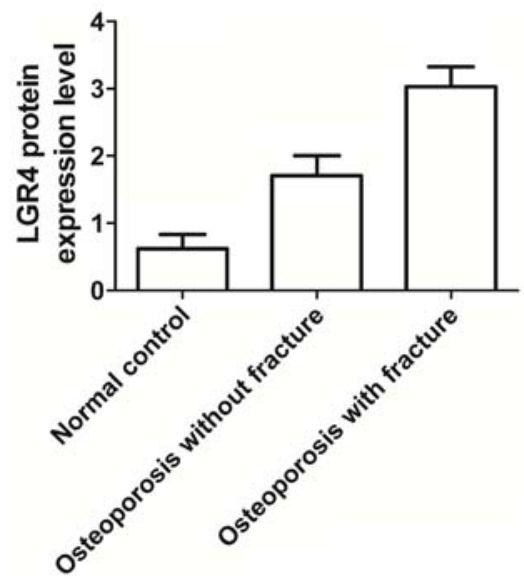

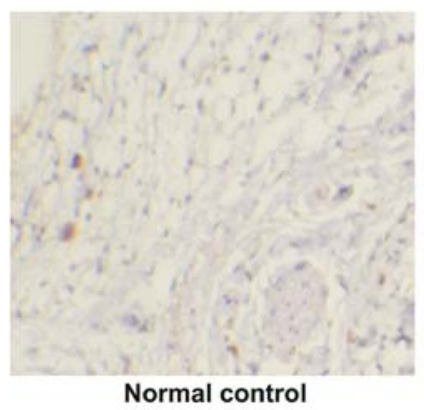

Normal control

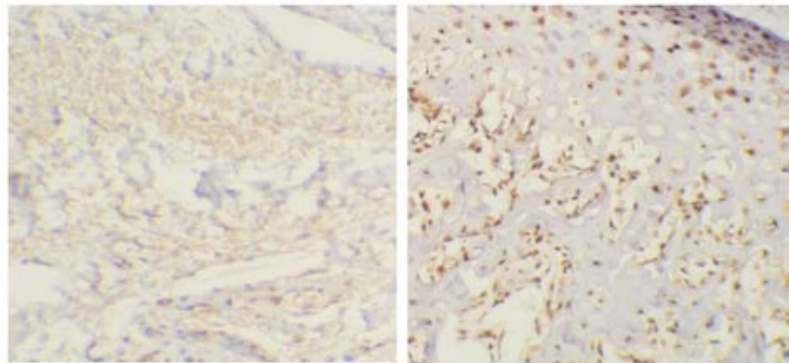

Osteoporosis without fracture
Osteoporosis with fracture

Figure 6. Real-time PCR and immunohistochemistry to detect the leucine-rich repeat-containing G-protein-coupled receptor 4 (LGR4) mRNA and protein among osteoporosis patients with fracture, osteoporosis patients without fracture and normal groups. (A) LGR4 mRNA was much higher in osteoporosis patients with fracture and osteoporosis patients without fracture than normal groups, while the LGR4 mRNA was significantly lower in osteoporosis patients with fracture group. (B) LGR4 protein was much higher in osteoporosis patients with fracture and osteoporosis patients without fracture than normal groups, while the LGR4 protein was significantly lower in osteoporosis patients with fracture group, as evaluated by immunohistochemistry. 

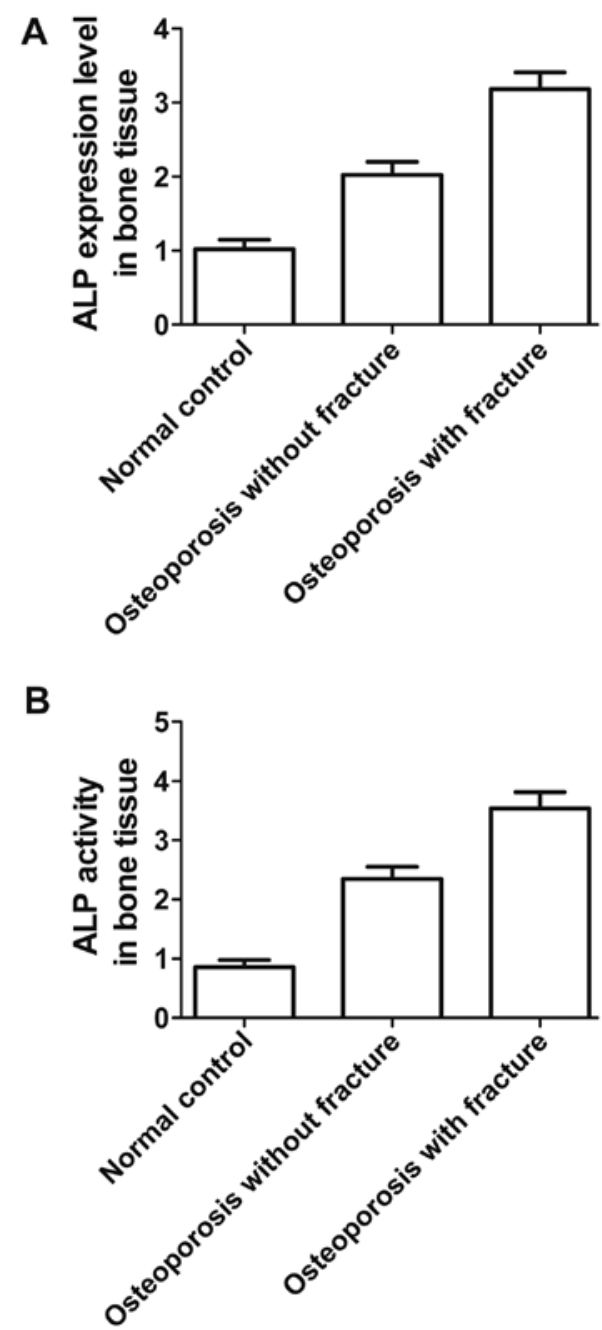

Figure 7. ELISA was used to detect the ALP level and ALP activity in bone tissue among osteoporosis patients with fracture, osteoporosis patients without fracture and normal groups. (A) ALP level was much higher in osteoporosis patients with fracture and osteoporosis patients without fracture than normal groups, while the ALP level was significantly lower in osteoporosis patients with fracture group. (B) ALP activity in bone tissue was much higher in osteoporosis patients with fracture and osteoporosis patients without fracture than normal groups, while the ALP activity were significantly lower in osteoporosis patients with fracture group.

that NSCLC cell proliferation and motility were inhibited by miR-137 directly through targeting of SLC22A18 (22). We found that NSCLC cell migration, invasion and proliferation could be inhibited by overexpression of miR-137 through the targeting of BMP7. As an important signaling molecule, bone morphogenetic protein (BMP) was first identified via its ability to induce the formation of cartilage and bone (23). As the expression of miR-137 is lost, so the ability of a cell to arrest at the $\mathrm{G} 1$ phase is reduced, which increases its proliferation, and thus may result in the accumulation of DNA damage which accordingly enhances genomic instability (24). In this study, we initially conducted realtime qPCR to estimate the expression of miR-137 among osteoporosis patients with and without fracture, and a normal group, and found that the expression was much lower in osteoporosis patients in both groups compared to normal patients, while the expression of miR-137 level was the lowest in the fracture group.
As another potential contributor, miR-137 plays a possible role in switching from proliferation to cellular differentiation (25).It has been reported that the expression level of miR-137 is reduced in poorly differentiated gliomas and increased in neuronal differentiation, although it is not currently known whether this generalizes to other histology (26). Conversely, because of our ability to detect such a relationship is limited by low statistical power, no relationship has been discovered between miR-137 promoter methylation and grade of tumor. Although it involves the control of cell cycle, no significant relationship between miR-137 promoter methylation and other squamous cell carcinoma of the head and neck (SCCHN) prognostic factors, such as tumor size, stage, surgical tumor margin positivity or nodal involvement has been found (27). In this study, we searched a miRNA online database (www. mirdb.org) to find the target gene of miR-137, and found that LGR4 was a virtual target of miR-137 with a potential binding site in the 3'UTR of LGR4. Furthermore, we performed a luciferase assay to confirm that LGR4 is a direct shared target of miR-137, and found that the luciferase activity of cells transfected with wild-type LGR4 was significantly downregulated compared with mutant-type LGR4 and the scramble control group, while luciferase activity in cells transfected with mutant 3'UTR of LGR4 was substantially downregulated compared with the scramble control.

An orphan receptor can be encoded with the LGR4 gene and it has been shown in genome-based studies that its genetic features are associated with low BMD and osteoporotic fractures (18). It has been reported that local cytokines negatively regulate the BMP-induced increase in expression of the LGR4 gene, which are important for bone formation in addition to TGF beta and FGF $(28,29)$. It was reported that for osteoporotic patients an orphan receptor was encoded with the LGR4 gene which is identified as a genetic determinant for bone mass. In osteoblastic cells, the effects of BMP on LGR4 expression were examined. During culture, the LGR4 gene was expressed in the osteoblastic cell line MC3T3E1 in a time-dependent manner. The expression of LGR4 mRNA was partially enhanced by BMP treatment via transcriptional events. While LGR4 mRNA was downregulated, the BMP-induced increase in ALP mRNA and ALP activity was inhibited. FGF suppressed BMP enhancement of LGR4 gene expression, but this was reversed by dexamethasone. In primary cultures of calvarial osteoblasts, the expression of LGR4 was also enhanced by BMP. It can be inferred from these data that $\mathrm{BMP}$ regulates the LGR4 gene which is required for BMP to influence osteoblastic differentiation (17).

LGR4 is located on chromosome 11 at position 11p14-p13, which is $\sim 107 \mathrm{~kb}$ in length. As one of the genes under active investigation in our laboratory, LGR4 is also known as $\mathrm{G}$ protein-coupled receptor 48 (Gpr48). LGR4 is widely expressed and critical for the development of many organs including kidneys, the reproductive tract, heart, cartilage, the nervous system and the intestines (30). LGR4-knockout mice demonstrated the importance of LGR4 on the development of various organs such as the male reproductive tract, kidneys, eyelids, erythropoiesis, hair placode, gallbladder and cystic duct, and bones (31-36). A recent study revealed a variant at position 126 caused by a c. $376 \mathrm{C}<\mathrm{T}$ nonsense mutation of human LGR4 (37). This mutation resulted in the complete loss 
of function of LGR4, and had important association with risks for osteoporosis, resulting in low BMD, gallbladder/biliary tract cancer, skin SCC and reduced testosterone levels (18).

As an end-point clinical outcome of osteoporosis, OF also has moderate heritability ranging from about 0.5 to 0.7 (8). We now know that GWASs and their meta-analyses have identified over 20 genes/loci related to risk of OF and more than 60 genes/loci related to variations in BMD. Moreover, a rare, novel, nonsense mutation within the LGR4 gene strongly related to low $\mathrm{BMD}$ and $\mathrm{OF}$ was identified in a recently published study involving whole-genome sequencing (18). In the present study, we performed real-time PCR and western blot analysis to evaluate LGR4 mRNA and protein in U-2 and MC3T3 cells in osteoporosis patients with and without fracture and normal patients, and found that LGR4 mRNA and protein levels in both cell types were much higher when transfected with miR-137 inhibitor, while LGR4 mRNA and protein levels were much lower following transfection with miR-137 mimics than the scramble control. Finally, we performed real-time PCR and immunohistochemistry to measure LGR4 mRNA and protein expression, and ALP expression and ALP activity in bone tissue in osteoporosis patients with and without fractures, and the normal group, and found that they were much higher in osteoporosis patients with and without fractures than the normal group, while they were significantly the lowest in osteoporosis patients with fractures.

In conclusion, these data prove that overexpression of miR-137 is associated with an altered risk of fracture in patients with osteoporosis, and could be recognized as a biomarker to predict risk of fracture in osteoporosis.

\section{Acknowledgements}

Not applicable.

\section{Funding}

No funding was received.

\section{Availability of data and material}

The datasets used and/or analyzed during the current study are available from the corresponding author on reasonable request.

\section{Authors' contributions}

XL planned the study, collected, analyzed and interpreted the data and prepared the manuscript. XH planned the study, collected the data, prepared the manuscript and analyzed the literature. Both authors read and approved the final manuscript.

\section{Ethics approval and consent to participate}

The institution's Ethics and Research Committees approved this study. The patients signed informed consent for participation in the study.

\section{Consent for publication}

Not applicable.

\section{Competing interests}

The authors declare that they have no competing interests.

\section{References}

1. Bruyère $\mathrm{O}$, Cooper C, Pelletier JP, Maheu E, Rannou F, Branco J, Luisa Brandi M, Kanis JA, Altman RD, Hochberg MC, et al: A consensus statement on the European Society for Clinical and Economic Aspects of Osteoporosis and Osteoarthritis (ESCEO) algorithm for the management of knee osteoarthritis - From evidence-based medicine to the real-life setting. Semin Arthritis Rheum 45 (Suppl 4): S3-S11, 2016.

2. Melton LJ III, Gabriel SE, Crowson CS, Tosteson AN, Johnell O and Kanis JA: Cost-equivalence of different osteoporotic fractures. Osteoporos Int 14: 383-388, 2003.

3. Adachi JD, Ioannidis G, Pickard L, Berger C, Prior JC, Joseph L, Hanley DA, Olszynski WP, Murray TM, Anastassiades T, et al: The association between osteoporotic fractures and health-related quality of life as measured by the Health Utilities Index in the Canadian Multicentre Osteoporosis Study (CaMos). Osteoporos Int 14: 895-904, 2003

4. Papaioannou A, Morin S, Cheung AM, Atkinson S, Brown JP, Feldman S, Hanley DA, Hodsman A, Jamal SA, Kaiser SM, et al; Scientific Advisory Council of Osteoporosis Canada: 2010 clinical practice guidelines for the diagnosis and management of osteoporosis in Canada: summary. CMAJ 182: 1864-1873, 2010.

5. Orimo H,Nakamura T, Hosoi T,Iki M,Uenishi K,Endo N,OhtaH, Shiraki M, Sugimoto T, Suzuki T, et al: Japanese 2011 guidelines for prevention and treatment of osteoporosis - executive summary. Arch Osteoporos 7: 3-20, 2012.

6. de Almeida Pereira Coutinho M, Bandeira E, de Almeida JM, Godoi ET, Vasconcelos G and Bandeira F: Low bone mass is associated with increased carotid intima media thickness in men with type 2 diabetes mellitus. Clin Med Insights Endocrinol Diabetes 6: 1-6, 2013

7. Ralston SH: Genetics of osteoporosis. Rev Endocr Metab Disord 2: 13-21, 2001.

8. Deng HW, Mahaney MC, Williams JT, Li J, Conway T, Davies KM, Li JL, Deng H and Recker RR: Relevance of the genes for bone mass variation to susceptibility to osteoporotic fractures and its implications to gene search for complex human diseases. Genet Epidemiol 22: 12-25, 2002.

9. Landgraf P, Rusu M, Sheridan R, Sewer A, Iovino N, Aravin A, Pfeffer S, Rice A, Kamphorst AO, Landthaler M, et al: A mammalian microRNA expression atlas based on small RNA library sequencing. Cell 129: 1401-1414, 2007.

10. Lee RC, Feinbaum RL and Ambros V: The C. elegans heterochronic gene lin-4 encodes small RNAs with antisense complementarity to lin-14. Cell 75: 843-854, 1993.

11. Kozomara A and Griffiths-Jones S: miRBase: annotating high confidence microRNAs using deep sequencing data. Nucleic Acids Res 42 (D1): D68-D73, 2014.

12. Pauley KM and Cha S: miRNA-146a in rheumatoid arthritis: a new therapeutic strategy. Immunotherapy 3: 829-831, 2011.

13. Cheng P, Chen C, He HB, Hu R, Zhou HD, Xie H, Zhu W, Dai RC, $\mathrm{Wu} \mathrm{XP}$, Liao EY, et al: miR-148a regulates osteoclastogenesis by targeting V-maf musculoaponeurotic fibrosarcoma oncogene homolog B. J Bone Miner Res 28: 1180-1190, 2013.

14. Wang Y, Li L, Moore BT, Peng XH, Fang X, Lappe JM, Recker RR and Xiao P: miR-133a in human circulating monocytes: a potential biomarker associated with postmenopausal osteoporosis. PLoS One 7: e34641, 2012.

15. Seeliger C, Karpinski K, Haug AT, Vester H, Schmitt A, Bauer JS and van Griensven M: Five freely circulating miRNAs and bone tissue miRNAs are associated with osteoporotic fractures. J Bone Miner Res 29: 1718-1728, 2014.

16. Garmilla-Ezquerra P, Sañudo C, Delgado-Calle J, PérezNuñez MI, Sumillera M and Riancho JA: Analysis of the bone microRNome in osteoporotic fractures. Calcif Tissue Int 96: 30-37, 2015.

17. Pawaputanon Na Mahasarakham C, Ezura Y, Kawasaki M, Smriti A, Moriya S, Yamada T, Izu Y, Nifuji A, Nishimori K, Izumi Y, et al: BMP-2 enhances Lgr4 gene expression in osteoblastic cells. J Cell Physiol 231: 887-895, 2016.

18. Styrkarsdottir U, Thorleifsson G, Sulem P, Gudbjartsson DF, Sigurdsson A, Jonasdottir A, Jonasdottir A, Oddsson A, Helgason A, Magnusson OT, et al: Nonsense mutation in the $L G R 4$ gene is associated with several human diseases and other traits. Nature 497: 517-520, 2013. 
19. Xiao J, Peng F, Yu C, Wang M, Li X, Li Z, Jiang J and Sun C: microRNA-137 modulates pancreatic cancer cells tumor growth, invasion and sensitivity to chemotherapy. Int J Clin Exp Pathol 7: 7442-7450, 2014

20. Hao S, Luo C, Abukiwan A, Wang G, He J, Huang L, Weber CE Lv N, Xiao X, Eichmüller SB, et al: miR-137 inhibits proliferation of melanoma cells by targeting PAK2. Exp Dermatol 24: 947-952, 2015.

21. Smith AR, Marquez RT, Tsao WC, Pathak S, Roy A, Ping J, Wilkerson B, Lan L, Meng W, Neufeld KL, et al: Tumor suppressive microRNA-137 negatively regulates Musashi-1 and colorectal cancer progression. Oncotarget 6: 12558-12573, 2015.

22. Zhang B, Liu T, Wu T, Wang Z, Rao Z and Gao J: MicroRNA-137 functions as a tumor suppressor in human non-small cell lung cancer by targeting SLC22A18. Int J Biol Macromol 74: 111-118, 2015.

23. Cheng H, Jiang W, Phillips FM, Haydon RC, Peng Y, Zhou L, Luu HH, An N, Breyer B, Vanichakarn P, et al: Osteogenic activity of the fourteen types of human bone morphogenetic proteins (BMPs). J Bone Joint Surg Am 85: 1544-1552, 2003

24. Kozaki K, Imoto I, Mogi S, Omura K and Inazawa J: Exploration of tumor-suppressive microRNAs silenced by DNA hypermethylation in oral cancer. Cancer Res 68: 2094-2105, 2008

25. Tarantino C, Paolella G, Cozzuto L, Minopoli G, Pastore L, Parisi S and Russo T: miRNA 34a, 100, and 137 modulate differentiation of mouse embryonic stem cells. FASEB J 24: 3255-3263, 2010.

26. Silber J, Lim DA, Petritsch C, Persson AI, Maunakea AK, Yu M Vandenberg SR, Ginzinger DG, James CD, Costello JF, et al: miR-124 and miR-137 inhibit proliferation of glioblastoma multiforme cells and induce differentiation of brain tumor stem cells. BMC Med 6: 14, 2008.

27. Langevin SM, Stone RA, Bunker CH, Lyons-Weiler MA LaFramboise WA, Kelly L, Seethala RR, Grandis JR, Sobol RW and Taioli E: MicroRNA-137 promoter methylation is associated with poorer overall survival in patients with squamous cell carcinoma of the head and neck. Cancer 117: 1454-1462, 2011.

28. Zhen $G$ and Cao X: Targeting TGF $\beta$ signaling in subchondral bone and articular cartilage homeostasis. Trends Pharmacol Sci 35: 227-236, 2014.
29. Itkin T, Kaufmann KB, Gur-Cohen S, Ludin A and Lapidot T: Fibroblast growth factor signaling promotes physiological bone remodeling and stem cell self-renewal. Curr Opin Hematol 20: 237-244, 2013

30. Du B, Luo W, Li R, Tan B, Han H, Lu X, Li D, Qian M, Zhang D, Zhao Y, et al: Lgr4/Gpr48 negatively regulates TLR2/4-associated pattern recognition and innate immunity by targeting CD14 expression. J Biol Chem 288: 15131-15141, 2013.

31. Mendive F, Laurent P, Van Schoore G, Skarnes W, Pochet R and Vassart G: Defective postnatal development of the male reproductive tract in LGR4 knockout mice. Dev Biol 290: 421-434, 2006.

32. Kato S, Matsubara M, Matsuo T, Mohri Y, Kazama I, Hatano R, Umezawa A and Nishimori K: Leucine-rich repeat-containing G protein-coupled receptor-4 (LGR4, Gpr48) is essential for renal development in mice. Nephron Exp Nephrol 104: e63-e75, 2006.

33. Kato S, Mohri Y, Matsuo T, Ogawa E, Umezawa A, Okuyama R and Nishimori K: Eye-open at birth phenotype with reduced keratinocyte motility in LGR4 null mice. FEBS Lett 581: 4685-4690, 2007.

34. Song H, Luo J, Luo W, Weng J, Wang Z, Li B, Li D and Liu M: Inactivation of G-protein-coupled receptor 48 (Gpr48/Lgr4) impairs definitive erythropoiesis at midgestation through down-regulation of the ATF4 signaling pathway. J Biol Chem 283: 36687-36697, 2008.

35. Mohri Y, Kato S, Umezawa A, Okuyama R and Nishimori K: Impaired hair placode formation with reduced expression of hair follicle-related genes in mice lacking Lgr4. Dev Dyn 237: 2235-2242, 2008.

36. Yamashita R, Takegawa Y, Sakumoto M, Nakahara M, Kawazu H, Hoshii T, Araki K, Yokouchi Y and Yamamura K: Defective development of the gall bladder and cystic duct in Lgr4- hypomorphic mice. Dev Dyn 238: 993-1000, 2009.

37. Luo J, Zhou W, Zhou X, Li D, Weng J, Yi Z, Cho SG, Li C, $\mathrm{Yi} \mathrm{T}, \mathrm{Wu} \mathrm{X}$, et al: Regulation of bone formation and remodeling by G-protein-coupled receptor 48. Development 136: 2747-2756, 2009. 